\title{
Kiss and run - phosphoglycerol modifications of N. meningitidis pili mediate detachment
}

\author{
Tone Tønjum ${ }^{1,2 *}$ and Michael Koomey ${ }^{1,3}$ \\ Centre for Molecular Biology and Neuroscience, University of Oslo, Oslo, Norway \\ 2 Department of Microbiology, Oslo University Hospital, Oslo, Norway \\ ${ }^{3}$ Department of Molecular Biosciences, University of Oslo, Oslo, Norway \\ *Correspondence: tone.tonjum@medisin.uio.no
}

\section{A commentary on}

Posttranslational Modification of Pili upon Cell Contact Triggers N. meningitidis dissemination

by Chamot-Rooke, J., Mikaty, G., Malosse, C., Soyer, M., Dumont, A., Gault, J., Imhaus, A. F., Martin, P., Trellet, M., Clary, G., Chafey, P., Camoin, L., Nilges, M., Nassif, X., and Duménil, G. (2011). Science 331, 778-782.

The bacterium Neisseria meningitidis normally colonizes the throat of $10-30 \%$ of the human population, but oro-pharyngeal colonization, not excluding other sites, can also act as the port of entry to the blood to cause septicemia, and then to the brain to cause meningitis. Colonization is primarily mediated by filamentous surface organelles entitled type IV pili, which allow the formation of bacterial aggregates associated with host cells.

Chamot-Rooke et al. (2011) report that contact of $N$. meningitidis with human cell triggers the expression of a posttranslational modification (PTM) system affecting pili, an event they associate with progressive detachment of bacteria from microcolonies attached to the cells. They hypothesize that this process may enable the bacteria to spread across tissue, facilitate invasion into epithelial tissue (thus promoting invasive disease), and perhaps promote transmission between human hosts.

Interestingly, Chamot-Rooke et al. (2011) observed that proliferation of $N$. meningitidis in contact with host cells increased the transcription of a bacterial gene encoding a putative phospho-form transferase (PptB) that adds phosphoglycerol (PG) onto the pilin subunit of type IV pili. The increase in $p p t B$ transcription was associated with an increase in the overall levels of pilin modification with this unique PTM, a result that could most easily be ascribed to altered microhetero- geneity with an increase in the number of residues bearing PG. They further report that inactivation of $p p t B$ and substitution of pilin serine residue 93 (a conserved and likely site of PG occupancy in strain 8013) led to an approximately two-fold increase in the number of bacteria found in aggregates. Likewise, overexpression of $p p t B$ resulted in a decrease in bacterial aggregation. As type IV pili are the predominant effectors of such bacterial aggregates, these results provided a connection between altered pilin modification with PG and a pilus - associated phenotype. In an effort to understand this association, molecular models of the threedimensional structure of pilin and pilus fibers were generated. Here, the data were amenable to the idea that PG site occupancy at serine 93 would negatively impact on lateral interactions between pili implicated in cellular aggregation. In line with this, induction of the de-repressible $p p t B$ allele also led to the abrogation in the expression of pili in their bundled or laterally aggregated form.

The authors also report that although the initial levels and overall levels of adherence to epithelial cells are statistically indistinguishable between the wildtype, $p p t B$, and pilin mutant carrying the serine to alanine substitution at residue 93 , shedding of detached bacteria was 3.5-fold diminished in the latter two strains relative to that of the former. As a consummating result, the authors report that the $p p t B$ and pilin mutants carrying the serine to alanine substitution at residue 93 are defective (20-fold) in the capacity to migrate across the epithelial cell monolayer.

Make no doubt about it, the work makes fascinating reading. The authors tout their findings as showing that "Through a regulated PTM, N. meningitidis adopts a multiply and-run strategy, presumably selected, and fine-tuned through evolution as a propagation mechanism, key for survival of the bacterium in nature." But to what degree do the findings made in vitro relate to the situation in vivo? And to what degree are the findings here consonant with those from other works?

One area of potential concern relates to the moderate up-regulation of $p p t B$ following contact with epithelial cells. This phenotype has been defined by comparisons of transcription profiles between bacteria growing in media versus growth in contact with epithelial cells, conditions that are convenient for study. However, it has yet to be substantiated that such alterations in transcription profiles occurs in vivo. Can we then be sure that modulation of $p p t B$ (or any other of the so-called contact regulated genes) occurs outside the lab?

Another area perhaps warranting scrutiny involves the fact that pilin in $N$. meningitidis is antigenically variable and undergoes dramatic changes in primary structure. Does the presence or absence of PG have the same effect on all such variants or is it limited to only a subset thereof? Or to put it another way, has pilin evolution been constrained so as to optimize the effects of PG modification, regulated, or otherwise? Both serine 69 and serine 93 are located in conserved domains of PilE, indicating a remarkable avoidance of variable regions.

Yet another specific finding made in the work is that the initial levels of bacterial attachment to epithelial cells were not altered by the constitutive expression of high levels of PG modification, although these strains failed to aggregate and expressed pili in an unbundled form. This result represents a potential sea change in the consensus view of the importance of aggregation on overall adherence. Specifically, an earlier study from the same group concluded that "aggregative (bundled) pili promote high adhesiveness of encapsulated meningococci" (Marceau et al., 1995). Furthermore, a study examining isogenic gonococcal variants carrying different pilin alleles observed a remarkable correlation between overall 
adherence levels and aggregation status (Park et al., 2001). Notably, the latter study also observed highly bundled pili in some non-aggregating and poorly adherent strains. Why such clear differences are seen in this article here versus those from the earlier works remains to be determined.

In summary, modifications of Type IV pili with the phospho-forms PG, phosphoethanolamine, and phosphocholine are undoubtedly of importance to bacterial persistence in the human host and the ecology of pathogenic and symbiotic Neisserial strains. It is particularly noteworthy that direct covalent modification with phosphoethanolamine has also been recently reported for an important protein component of flagella in Campylobacter jejuni (Cullen and Trent, 2010). Thus, these modifications can hardly be regarded as mere decorations or curiosities of Neisserial biology.
Being able to understand how and why pili are decorated in a certain manner during the distinct phases of infection will facilitate the understanding of pathogenesis and symbiosis. These PTMs are also highly relevant for eukaryotic systems. The work presented clearly provides an interesting insight as to how pilin PG modification might function in N. meningitides, as a significant contribution to a long story. Stay tuned.

\section{REFERENCES}

Chamot-Rooke, J., Mikaty, G., Malosse, C., Soyer, M., Dumont, A., Gault, J., Imhaus, A. F., Martin, P., Trellet, M., Clary, G., Chafey, P., Camoin, L., Nilges, M., Nassif, X., and Duménil, G. (2011). Posttranslational modification of pili upon cell contact triggers $N$. meningitidis dissemination. Science 331, 778-782.

Cullen, T. W., and Trent, M. S. (2010). A link between the assembly of flagella and lipooligosaccharide of the Gram-negative bacterium Campylobacter jejuni. Proc. Natl. Acad. Sci. U. S. A. 107, 5160-5165.
Marceau, M., Beretti, J. L., and Nassif, X. (1995). High adhesiveness of encapsulated Neisseria meningitidis to epithelial cells is associated with the formation of bundles of pili. Mol. Microbiol. 17, 855-863.

Park, H. S., Wolfgang, M., van Putten, J. P., Dorward, D., Hayes, S. F., and Koomey, M. (2001). Structural alterations in a type IV pilus subunit protein result in concurrent defects in multicellular behaviour and adherence to host tissue. Mol. Microbiol.42,293-307.

Received:07 April 2011; accepted:07 April 2011; published online: 27 April 2011.

Citation: Tønjum T and Koomey M (2011) Kiss and run - phosphoglycerol modifications of $N$. meningitidis pili mediate detachment. Front. Microbio. 2:82. doi: 10.3389/ fmicb.2011.00082

This article was submitted to Frontiers in Cellular and Infection Microbiology, a specialty of Frontiers in Microbiology.

Copyright (C) 2011 Tønjum and Koomey. This is an openaccess article subject to a non-exclusive license between the authors and Frontiers Media SA, which permits use, distribution and reproduction in other forums, provided the original authors and source are credited and other Frontiers conditions are complied with. 